\title{
Efeito da Intervenção Psicológica Vocacional na Indecisão e Comportamento Exploratório
}

\author{
Career Counseling Effects on Indecision \\ and Exploratory Behavior
}

Efecto de la Intervención Psicológica Vocacional en la Indecisión y Comportamiento Exploratorio

Maria Céu Taveira Castro Silva Brás Cunha \& Liliana da Costa Faria

Universidade do Minho - Portugal
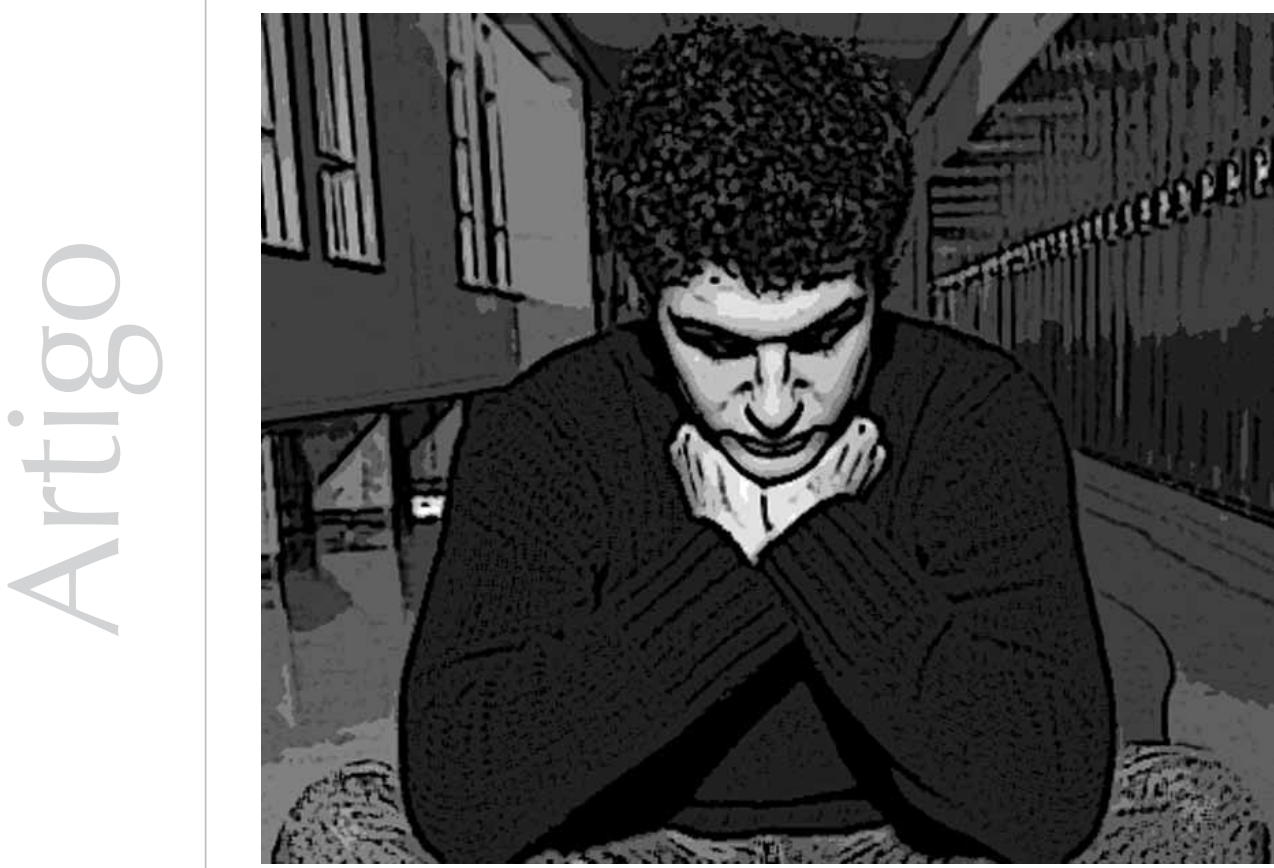
Resumo: Um adolescente veio à consulta psicológica com o objectivo de receber ajuda para um problema de indecisão face à vida escolar e profissional. Os dados da avaliação indicam como causas do problema a falta de consciência de si próprio, o desconhecimento sobre o processo de tomada de decisão, um fraco conhecimento do mundo escolar e profissional e dificuldades no compromisso com um curso de acção. O cliente apresenta crenças de carreira negativas, pensamentos ansiosos face ao futuro, conduta exploratória pouco intencional e sistemática e indecisão. As recomendações terapêuticas consistiram em seguir um programa de tratamento psicológico, ao longo de cinco sessões, com o objectivo de mudar os pensamentos negativos e favorecer os níveis de exploração e de compromisso do cliente. Após as cinco sessões de tratamento, registra-se um aumento das crenças e dos comportamentos positivos de exploração e uma redução dos níveis de ansiedade exploratória e de decisão, bem como uma redução dos níveis de indecisão. Conclui-se acerca dos efeitos positivos da consulta psicológica breve e estruturada na tomada de decisão vocacional.

Palavras-chave: Tomada de decisão. Comportamento exploratório. Intervenção psicológica. Estudo de caso.

\begin{abstract}
An adolescent came to psychological counselling in order to obtain help for a career indecision problem. Assessment data indicate as problem causes the lack of self-awareness, the absence of knowledge about the career decision-making process, poor understanding of the academic and professional world and difficulties in the commitment with a plan of action. The client displays negative career beliefs, anxious thoughts about the future, unintentional and unsystematic exploratory behaviour and career indecision. A psychological treatment program, composed by five sessions, was followed, in order to change the negative beliefs and to enhance the client exploration and commitment about the career. After the five treatment sessions, there was an increase in positive career exploration beliefs and behaviours, and a decrease in the anxiety levels related to the exploration and decision-making processes as well as a decline in the career indecision levels. Furthermore, one can conclude about the benefits of brief and structured psychological counselling in career decision-making.
\end{abstract}

Keywords: Career decision making. Exploratory behaviour. Psychological intervention. Case study.

Resumen: Un adolescente vino a la consulta psicológica con el objetivo de recibir ayuda para un problema de indecisión de cara a la vida escolar y profesional. Los datos de la evaluación indican como causas del problema la falta de conciencia de sí mismo, el desconocimiento sobre el proceso de tomada de decisión, un flojo conocimiento del mundo escolar y profesional y dificultades en el compromiso con un curso de acción. El cliente presenta creencias de carrera negativas, pensamientos ansiosos de cara al porvenir, conducta exploratoria poco intencional y sistemática e indecisión. Las recomendaciones terapéuticas consistieron en seguir un programa de tratamiento psicológico, a lo largo de cinco sesiones, con el objetivo de alterar los pensamientos negativos y favorecer los niveles de exploración y de compromiso del cliente. Después de las cinco sesiones de tratamiento, se registra un aumento de las creencias y de los comportamientos positivos de exploración y una reducción de los niveles de ansiedad exploratoria y de decisión, así como una reducción de los niveles de indecisión. Se concluye acerca de los efectos positivos de la consulta psicológica breve y estructurada en la toma de decisión vocacional.

Palabras clave: Toma de decisión. Comportamiento exploratorio. Intervención psicológica. Estudio de caso.

O estabelecimento de compromissos face à vida escolar e profissional constitui um indicador do desenvolvimento positivo na adolescência e um precursor da capacidade de lidar com desafios e adversidades em fases posteriores da carreira (Lent \& Brown, 1996). Nesse âmbito, a realização de escolhas face a papéis de vida é um elemento crucial do comportamento humano (Silva, 1997) e também uma problemática vocacional (Rounds \& Tinsley, 1984). A escolha vocacional consiste, em geral, em uma resposta verbal, que envolve uma mudança directa ou indirectamente observável do comportamento (e.g., escrever, falar, procurar e avaliar informação), relacionada com a vida pessoal e profissional futuras (Crites, 1969).

Assim, por exemplo, um adolescente, quando questionado acerca do seu futuro, pode afirmar: "Vou escolher o curso de ciências no ensino fundamental porque mais tarde quero freqüentar o curso de Medicina, vir a ser psiquiatra numa clínica, ter a minha própria 
casa e viajar". Nesse sentido, a escolha vocacional pode ser a acção de eleger, em um dado momento, uma alternativa e iniciar um plano de execução de objectivos de curto prazo, ligados a uma cadeia de objectivos mais amplos e de longo prazo (Germeijs \& De Boeck, 2003; Rassin \& Muris, 2005).

Para isso, são necessárias, como nos evidencia Silva (1997), as seguintes três condições básicas (Crites, 1969): (a) o suprimento da escolha - a existência de pelo menos duas opções alternativas de escolha, percebidas como prováveis; (b) o incentivo - a motivação para realizar a escolha e (c) a liberdade de escolha - poder optar livremente por uma via, papel ou actividade. Mesmo garantidas essas condições básicas, podem ser observadas dificuldades nas escolhas face ao futuro, derivadas de fracas oportunidades de desenvolvimento pessoal e vocacional, fraco aproveitamento dessas mesmas oportunidades pelos sujeitos ou por influência negativa de determinadas experiências sociais e culturais (Taveira, 2001).

Desde os anos 50 do século passado, um vasto corpo de investigadores tem procurado compreender as múltiplas causas e factores das dificuldades das pessoas em decidir sobre o tipo de trabalho a prosseguir em um dado momento da vida (Gati, 2006; Miller \& Brown, 2005). A falta de conhecimento, a ansiedade, a instabilidade dos objectivos de vida, a baixa auto-eficácia na tomada de decisão de carreira, bem como os níveis baixos de maturidade vocacional e os modos menos adaptativos de construção da identidade vocacional, são alguns dos factores de indecisão estudados e relacionados com esquemas de diagnóstico e de intervenção específicos (Armstrong \& Rounds, 2008; Chartrand \& Robbins, 1997).
Diversos autores procuram estudar agrupamentos homogéneos de clientes indecisos, tendo identificado dois tipos de clientes no que respeita a indecisão vocacional: os indecisos desenvolvimentais e os indecisos crónicos. Ambos serão brevemente descritos a seguir.

Os primeiros necessitam de informação sobre o self e sobre as ocupações. Os segundos tendem a apresentar indecisão face a uma variedade de desafios ou actividades de vida, tais como ansiedade traço e afectividade negativa, uma tendência para a focagem e ruminação acerca do problema, rejeição de várias escolhas por medo do compromisso, percepção de baixos níveis de capacidade de resolução do problema, crenças negativas de auto-eficácia na tomada de decisão e percepções de fraca estabilidade dos seus objectivos (Brown \& McPartland, 2005; Peterson, Sampson, Lenz, \& Reardon, 2002).

Os indecisos desenvolvimentais respondem, normalmente, bastante bem à consulta psicológica breve, enquanto os indecisos crónicos requerem uma intervenção psicológica mais longa, até conseguirem chegar a uma decisão (Whiston, Brecheisen, \& Stephens, 2003). O uso da análise de clusters permitiu identificar outros dois subtipos de clientes: os que experienciam ansiedade apenas focada no problema actual devido, por exemplo, à eminência da decisão (Chartrand \& Robbins, 1997) e aqueles que se sentem paralisados devido a conflitos com outros significativos no que respeita a preferência por uma escolha (Kelly \& Lee, 2002).

Os primeiros podem necessitar de gerir a sua ansiedade e encontrar uma direcção viável, enquanto os segundos podem necessitar de ajuda não tanto para explorar opções viáveis, 
O conhecimento de si e do meio ocorrerá através da exploração que os adolescentes forem capazes de realizar.

Esta tem sido entendida como "o processo psicológico que sustenta as actividades de procura e de processamento de informação e o teste de hipóteses sobre o self e o meio" (Taveira, 1997. p. 3). mas mais para saber gerir o conflito envolvido na decisão. Este requer, muitas vezes, a capacidade para captar apoio incondicional de outros significativos para os seus planos e abertura para trabalhar o compromisso com opções.

A consulta psicológica individual pode ser uma das modalidades de ajuda mais efectivas, nesses casos. Outros clientes podem estar indecisos e confusos devido à percepção de múltiplas escolhas viáveis (Gati, Kraus, \& Osipow, 1996), enquanto outros, ainda, podem estar indecisos vocacionalmente porque têm que escolher entre um leque muito restrito de opções (Shimizu, Vondracek, Schulenberg, \& Hjostetler, 1988); finalmente, outros podem estar indecisos porque necessitam de uma validação externa da sua escolha, antes mesmo de se comprometerem publicamente com essa decisão (Chartrand, Robbins, \& Morrill, 1994).

Essa linha de estudo é importante, tanto mais que as dificuldades na tomada de decisão de carreira podem afectar, por exemplo, os níveis de exploração vocacional e, em conseqüência, outros resultados de carreira relevantes, como o desenvolvimento do autoconceito, os níveis de auto-eficácia na tomada de decisão, o valor atribuído aos objectivos escolares e profissionais (Stumpf, Collarelli, \& Hartman, 1983) ou mesmo o ajustamento a uma escolha de carreira, à maturidade vocacional e à satisfação profissional (Taveira, 2000; Veerle, Karine, \& Bart, 2006). Nesse sentido, intervir nos problemas de decisão implica, primeiramente, ajudar os adolescentes a conhecerem-se a si próprios e ao meio bem como aos sistemas de oportunidades vocacionais, de modo a realizar opções de carreira planeadas e consistentes, que possam concretizar e expandir interesses, valores, capacidades e aspirações pessoais (Afonso \& Taveira, 2001). O conhecimento de si e do meio ocorrerá através da exploração que os adolescentes forem capazes de realizar. Esta tem sido entendida como "o processo psicológico que sustenta as actividades de procura e de processamento de informação e o teste de hipóteses sobre o self e o meio" (Taveira, 1997, p. 3).

Nessa perspectiva, a conduta exploratória envolve experimentar, investigar, fazer tentativas e ensaios, procurar e questionar, perspectivar e testar hipóteses (Taveira, 2005). Krumboltz e Thoresen (1964) argumentam que a conduta exploratória instrumental (os comportamentos de procura de informação) pode ser necessária para reduzir a incerteza quanto à escolha de uma opção de carreira. A exploração vocacional tem sido reconhecida, também, como um componente importante do processo de apoio à decisão vocacional (Taveira, 1997). Nesse âmbito, é especialmente importante atender à qualidade da informação e não só à quantidade da informação que a pessoa explora (Greenhaus \& Connolly, 1982; Sugalsky \& Greenhaus, 1986) e ter em conta que a satisfação com a informação resultante da conduta exploratória bem como a ansiedade ou o stress antecipados face à idéia de explorar ou de decidir são efeitos da exploração com impacto significativo na exploração e no compromisso subseqüentes (Taveira, 2000; Veerle et al., 2006).

A resolução das tarefas de exploração vocacional orientada para si e para o meio e o compromisso com uma opção de carreira, no final do ensino obrigatório, têm sido associados a resultados positivos no desenvolvimento e na realização de escolhas posteriores (e.g., Jordan \& Super, 1974), o que faz com que os processos de exploração 
vocacional e de tomada de decisão sejam considerados factores protectores do processo de transição para a vida adulta (Erickon, 1968; Savickas, 1985). Assim, assume-se que a exploração seja simultaneamente um antecedente e um resultado importante da indecisão vocacional e da aprendizagem e desenvolvimento humanos (Berlyne, 1960; Bowlby, 1969; Erikson, 1968; Super, 1942).

A modalidade de intervenção considerada de excelência na resolução de problemas de indecisão de carreira é a consulta psicológica (Brown \& Krane, 2000). A eficácia da consulta psicológica está bem documentada em estudos meta-analíticos (Brown \& Krane, 2000; Oliver \& Spokane, 1988; Spokane \& Oliver, 1983; Whiston, Sexton, \& Lasoff, 1998).

Essa linha de estudo demonstrou que a magnitude do efeito total da consulta psicológica $(d)$ foi de 0.34 . Esse resultado significa que a média dos clientes com indecisão atendidos naquela modalidade, no final da intervenção, fica cerca de um terço de um desvio-padrão mais decididos, satisfeitos e seguros acerca das suas escolhas do que as pessoas que não receberam qualquer ajuda. Apesar desse valor de magnitude do efeito ser considerado modesto por certos autores (Cohen, 1988), outros (Brown \& McPartland, 2005) consideram-no significativo em termos sociais e práticos.

Os valores moderados de magnitude de efeito alcançados pela maioria das referidas intervenções poderão estar relacionados com o facto de a maioria das intervenções na indecisão vocacional se basearem ainda no modelo de aconselhamento de Parsons (1909), ou em teorias da carreira de traço-e-factor (Holland, 1997), focadas no diagnóstico, informação e estabelecimento de correspondências entre a pessoa e os ambientes vocacionais. Rounds (1990) demonstra, por exemplo, que uma combinação da congruência de interesses com a correspondência entre necessidades e recompensas tem muito mais valor preditivo dos níveis de satisfação com o trabalho do que a congruência ou a correspondência sozinhas. Porque um dos objectivos das intervenções de carreira para apoiar a decisão é ajudar os clientes a prever e a escolher ocupações que Ihes trarão satisfação, existe suporte teórico e empírico para utilizar tanto os interesses como os dados das necessidades e os valores para gerar possibilidades e exercitar a autocompreensão.

A avaliação das capacidades, por sua vez, parece ser, também, crucial, porque facilita a tomada de decisão dos mais talentosos intelectualmente (Archer \& Lubinsky, 2003). Mas todos os clientes podem beneficiar-se dela, como sugere a teoria do processamento de informação (Peterson et al., 2002). Devemos ajudar as pessoas a identificar talentos, a ultrapassar as influências limitadoras da carreira e a atingir o máximo a que os seus talentos podem levá-los, utilizando os melhores métodos existentes, e não evitandoos. Assim, o uso de testes como forma de avaliar as capacidades pode ser de grande valia na consulta psicológica, contribuindo para a expansão de possibilidades.

Outras teorias da carreira, como a teoria das aspirações vocacionais de Linda Gottfredson (1981), sugerem que algumas pessoas podem eliminar prematuramente opções de carreira viáveis porque têm crenças de auto-eficácia inadequadas, imprecisas, pouca informação, ou sofreram processos de socialização ligados ao sexo, e que é importante ajudar as pessoas durante a consulta psicológica a identificar e a reconsiderar tais possibilidades. 
Savickas (1996), por sua vez, na sua teoria da construção da carreira, advoga que uma compreensão mais completa da saliência do papel de trabalhador face a outros papéis de vida, a prontidão para tomar decisões e os temas de carreira devem ser avaliados, pois oferecem possibilidades de autodefinição valiosas que ajudam a tomar decisões mais informadas e esclarecidas.

Os estudos de meta-análise sugerem igualmente diversas idéias para aumentar a eficácia das intervenções. Brown e Krane (2000), por exemplo, identificam cinco estratégias que, quando empregadas nas intervenções para diminuir a indecisão de carreira, podem aumentar os resultados através de diferentes clientes e modalidades de intervenção, como é o caso da consulta psicológica. Essas estratégias são: (a) levar os clientes a estabelecer e a escrever objectivos relacionados com o seu trabalho futuro; (b) falar individualmente com os clientes sobre os objectivos de futuro estabelecidos; (c) expor os clientes a modelos efectivos de tomada de decisão; (d) ajudar os clientes a explorar informação ocupacional e a promover o uso de fontes de informação variadas entre as sessões; (e) ajudar os clientes a darem atenção, a identificarem e a construírem apoio para os seus planos preferidos de carreira (Whiston et al., 2003). O estudo de Brown e Krane (2000) demonstrou também que o uso combinado de quaisquer três desses cinco elementos, independentemente da teoria em que se baseia a intervenção, aumenta a magnitude do efeito das intervenções $(d=.99)$.

Como podemos verificar, a investigação oferece importantes dados sobre a eficácia das intervenções de carreira e alguns insights sobre o que pode ser estruturado para aumentar os seus benefícios. Existe evidência para afirmar que as intervenções nas dificuldades na tomada de decisão são eficazes, e que essa eficácia, ainda que modesta, pode ser aumentada de muitos modos.

Porque os dados agregados através de diversas pessoas ofuscam, freqüentemente, o modo como os indivíduos mudam, a investigação dos mecanismos de mudança individual é crucial (Hill \& Carter, 1983). Assim, propomos neste estudo descrever o resultado de um caso único de consulta psicológica destinada a apoiar dificuldades na tomada de decisão vocacional, usando medidas de resultado que incluem a indecisão e a exploração de carreira. O presente estudo segue a classificação das metodologias de investigação propostas por Montero e León (2002), bem como as normas para a redacção de casos clínicos de Buela-Casal e Sierra (2002).

\section{Método}

\section{Identificação do cliente}

Rodrigo (pseudónimo), 14 anos, sexo masculino, solteiro, estudante do 9 o ano de escolaridade ${ }^{1}$ em uma escola privada da região noroeste de Portugal, proveniente de uma família de nível socioeconómico e cultural de nível médio alto. O Rodrigo vive com os pais, com quem mantém uma boa relação, e com os quais fala sobre o futuro escolar e profissional com regularidade. Os seus pais são descritos como apoiantes, não pressionando o cliente a escolher um curso específico. Aspira no futuro ingressar no ensino superior, achando-se capaz de o conseguir.

Os pais também têm expectativas de que o filho faça um curso de ensino superior. Rodrigo é um adolescente bastante autónomo, revelando possuir capacidade de reflexão, 
referindo-se explicitamente ao fato de "que se encontra preocupado com a sua orientação vocacional". Afirmou mesmo que uma das razões para procurar ajuda psicológica se prende ao desconforto sentido por "estar a estudar não sabe bem para quê". Está preocupado com o facto de não ter um objectivo de carreira definido, nem uma meta específica que o faça sentir-se orientado no seu percurso académico. Nunca foi alvo de tratamento psicológico, sendo essa a primeira vez que recorre ao aconselhamento especializado.

\section{Identificação do psicólogo}

A psicóloga, Dra. A., tem 26 anos de idade, é licenciada em Psicologia e freqüenta um curso de doutoramento em Psicologia vocacional. Além disso, conta com três anos de experiência profissional no âmbito da consulta psicológica vocacional.

\section{Análise do motivo da consulta}

O cliente chega à consulta com um pedido de ajuda relacionado com a decisão que deve tomar no final do 9 o ano de escolaridade, ou seja, no final da escolaridade obrigatória. Sente-se indeciso quanto ao percurso escolar a prosseguir no ensino secundário ${ }^{2}$. A indecisão tem sido acompanhada por sintomas como ansiedade, forte questionamento aos pais, professores e colegas, e impaciência face à exploração das alternativas que o sistema de ensino e o mercado de trabalho oferecem após a escolaridade obrigatória.

${ }^{1} \mathrm{O}$ correspondente ao último ano do ensino fundamental no Brasil.

${ }^{2} \mathrm{O}$ correspondente ao ensino médio no Brasil.

de ajuda para um problema de indecisão de carreira. Essa indecisão relativa ao futuro escolar está a causar-lhe inquietação, repercutindo-se negativamente no seu dia consciente do seu problema e da importância de receber ajuda psicológica para solucionar as suas dificuldades; como tal, está motivado para a intervenção.

\section{História do problema}

O problema teve início no início do 9o ano de escolaridade, no ano lectivo de 2004/2005. Pela primeira vez, o Rodrigo começou a tomar consciência da necessidade de tomar uma decisão sobre a carreira no final do ano lectivo. Seria confrontado com uma escolha, e não sabia o que escolher. À medida que se foi aproximando o final do ano lectivo, a antecipação de que seria chamado a tomar uma decisão agudizou o problema, e o cliente começou a desenvolver sintomatologia ansiosa.

No princípio, o cliente não considerava o problema como inadaptação, uma vez que muitos dos seus amigos também expressavam não saber ainda que curso iriam seguir no final da escolaridade obrigatória. Com o passar do tempo, começou a ter pensamentos negativos relacionados com a decisão de carreira que teria que tomar. Sentia medo de escolher um curso que não fosse o mais adequado para si, em termos das suas competências, interesses e saídas profissionais.

\section{Análise e descrição dos comportamentos-problema}

As principais condutas-problema do cliente são a indecisão e a ansiedade na tomada de decisão. Estas, por sua vez, dividem-se em quatro componentes: cognitiva, fisiológica, emocional e comportamental. a dia. Há que referir que o cliente está
Na componente cognitiva, o Rodrigo apresenta um discurso autoderrotista, como resultado de disfuncionamento cognitivo (crenças negativas 
acerca das possibilidades gerais de emprego na área preferida e da possibilidade pessoal de conseguir atingir uma posição favorável no mercado de trabalho), que mantêm o problema e que geram maior ansiedade, quer na exploração vocacional, quer na tomada de decisão, por comparação a outros acontecimentos de vida (Gysbers \& Moore, 1987; Yost \& Corbishley, 1987).

Em nível fisiológico, o adolescente evidencia sintomas tais como: taquicardia, agitação e respiração acelerada quando é indagado pelos pais, professores, grupo de pares e outros sobre o seu futuro escolar e profissional a curto, médio e longo prazo. Em termos emocionais, o Rodrigo evidencia insatisfação face à informação obtida sobre as profissões, empregos e organizações mais relacionadas com os seus interesses, capacidades e aspirações. Por último, em nível comportamental, verifica-se uma baixa exploração intencional e sistemática de profissões, empregos e organizações de trabalho, por parte do Rodrigo, bem como uma baixa exploração intencional e sistemática dos seus interesses face a tais ocupações. Nota-se, ainda, a existência de informação inadequada, contraditória e/ou insuficiente.

\section{Estabelecimento das metas do tratamento}

As metas que se estabeleceram e se negociaram com o cliente para conseguir solucionar os seus problemas de indecisão e de ansiedade foram respectivamente: (a) esclarecer o cliente acerca da natureza da decisão vocacional a realizar; (b) apoiar o cliente no processo de (in)decisão sobre o futuro próximo; (c) ajudar o cliente a inscrever essa tarefa vocacional em uma visão mais abrangente da vida/carreira; (d) apoiar o diálogo e a comunicação entre o cliente e outros significativos a esse respeito e (e) antecipar possíveis dificuldades e soluções para lidar com a transição vocacional.

Em termos mais gerais, estipula-se como importante promover as crenças e os comportamentos de exploração bem como reduzir a ansiedade na exploração e na tomada de decisão, isto é, pretende-se diminuir as crenças negativas e os estados emocionais associados a estas bem como implementar condutas exploratórias.

\section{Estudo dos objectivos terapêuticos}

Para alcançar as metas de tratamento, é necessário intervir sobre as condutas objecto. Tendo em conta os objectivos terapêuticos, as condutas objecto de tratamento são as seguintes: (a) baixar os níveis de ansiedade para que o cliente consiga mais tranqüilidade; (b) mudar, na medida do possível, as crenças menos adaptativas por crenças mais adaptativas e funcionais; (c) promover o comportamento sistemático e intencional de exploração de si e do meio; (d) promover a aprendizagem do processo de tomada de decisão; (e) favorecer o compromisso com uma decisão e um plano de carreira e (f) prever os passos e apoios necessários ao sucesso na concretização da escolha/plano.

\section{Selecção do tratamento mais adequado}

A escolha do tratamento relacionou-se com dois factores principais, a fase de desenvolvimento psicossocial em que o cliente se encontra, a adolescência e o problema apresentado pelo cliente, a indecisão quanto à escolha de um curso de estudos no final da escolaridade obrigatória. Do ponto de vista desenvolvimental, o cliente, supostamente, encontra-se em uma fase da vida propícia à exploração vocacional, isto é, 
à exploração pessoal e do meio em termos do eu vocacional e de carreira (Savickas, 2002).

Durante essa fase, é previsto que o adolescente explore o mundo em que vive, a subcultura a que pertence, assim como os papéis que espera vir a desempenhar e que vão de encontro à sua personalidade, valores, aptidões e interesses, desenvolvendo e implementando os seus auto-conceitos (Taveira, 2000). Nesse sentido, espera-se que o adolescente assuma uma postura mais activa e reflexiva face a si mesmo e ao mundo, em uma busca de identificação pessoal, profissional e social.

Teoricamente, encontra-se a viver um período designado de tentativa, isto é, uma fase de transição entre a fantasia e a perspectiva realista de si mesmo e do mundo, à medida que se exige mais responsabilidade, autonomia e capacidade decisional, quer por modelagem social, quer também por necessidade de independência (Ginzberg, 1951). Essa etapa é constituída por marcos fundamentais, como o desenvolvimento de interesses, da consciência das suas capacidades, da consolidação de valores e do desenvolvimento de capacidades de tomada de decisão face às sucessivas transições a que está sujeito.

O desenvolvimento cognitivo, nomeadamente, a capacidade de raciocínio abstracto e hipotético-dedutivo, contribuem substancialmente para o desenvolvimento geral do adolescente, proporcionando oportunidades de praticar e desenvolver a sua perspectiva temporal de futuro ou o ensaio de papéis sociais, em imaginação e na realidade (Afonso, 2000; Taveira, 1997). O ensaio de papéis constitui fator crucial na consolidação da consciência das características pessoais e do mundo de trabalho (Super, 1957).
No caso do Rodrigo, ele é um adolescente com um autoconceito bem desenvolvido, consciente das suas capacidades e competências sociais, profissionais e escolares. Tem interesses definidos, está consciente da necessidade de tomar decisões de carreira e da importância dessas decisões para o seu futuro escolar e profissional, apresentando, pela sua história pessoal e motivo de consulta presente, uma clara preocupação em definir e planear a sua carreira, a curto e a longo prazo.

Tendo em conta o supra referido, o tratamento com o Rodrigo, no contexto da consulta psicológica, passa por trabalhar a cristalização de preferências (diferenciação e clarificação de interesses e valores pessoais, a formulação de preferências, aquisição de informação sobre as mesmas e planejamento da opção preferida de carreira), o que permitirá, por sua vez, incrementar a sua maturidade vocacional. Procurar-se-á ajudar o cliente a definir um leque de opções prováveis (Gottfredson, 1981), em que poderá compilar as opções seleccionadas, tendo por base critérios de selecção muitas vezes inconscientes, como, por exemplo, o sexo, a educação, o autoconceito e o prestígio percebido. Procurar-se-á fornecer mais meios de exploração de informação para que o cliente, com base no seu autoconhecimento e nos apoios de que dispõe, formule objectivos vocacionais mais sólidos e definidos.

O tratamento seleccionado seguiu a abordagem desenvolvimentista relacional do processo da consulta psicológica vocacional proposta por Taveira (2001, 2005), a partir dos modelos de Spokane (1991) e de Gibson e Mitchell (1998). O processo de tratamento estruturouse em quatro fases principais - iniciar, explorar, comprometer, finalizar-, cada uma das quais envolvendo o prosseguimento de intenções e técnicas mais específicas, da parte do psicólogo, e expectativas quanto a 
reacções mais específicas da parte do cliente (Gibson \& Mitchell, 1998; Spokane, 1991).

\section{Selecção e aplicação das} técnicas de avaliação e análise dos resultados obtidos nesta fase

As técnicas de avaliação foram seleccionadas em função das características do cliente, do problema e do tratamento seleccionado (Buela-Casal \& Sánchez, 2002; Buela-Casal \& Sierra, 2001). Os instrumentos de medida seleccionados para a avaliação psicológica do tratamento foram os seguintes: (a) a Career Decision Scale (CDS; Osipow, Carney, Winer, Yanico, \& Koschier, 1976), para avaliar as dimensões dos construtos psicológicos da tomada de decisão, nomeadamente, a indecisão de carreira, e (b) a Career Exploration Survey (CES, Stumpf et al., 1983), para avaliar o processo de exploração vocacional.

A versão da Career Decision Scale utilizada neste estudo, adaptada por Taveira (1997), destina-se a estudantes do 9 o ao $12^{\circ}$ anos de escolaridade. O estudo de adaptação dessa escala demonstra que a maioria dos seus itens apresenta boas qualidades metrológicas. É constituída por 15 itens, 14 dos quais (itens 1 a 14) cotados em uma escala de resposta tipo likert, com quatro categorias, em um formato gráfico-numérico em cada uma das alternativas de resposta. O item 15 é de resposta aberta, para que o participante apresente informação detalhada sobre o seu estado de (in)decisão, caso nenhuma das situações referidas nos 14 itens se lhe apliquem. O resultado final é obtido através de uma nota global da escala para medir a indecisão de carreira. O item 15 é interpretado qualitativamente (Taveira, 1997).

A versão da Career Exploration Survey utilizada neste estudo, desenvolvida por
Stumpf et al. (1983) e adaptada por Taveira, destina-se aos estudantes do $9^{\circ}$ e $12^{\circ}$ anos de escolaridade. É uma escala constituída por 54 itens, que permitem avaliar de modo consistente quatro tipos de crenças, cinco tipos de comportamentos e três tipos de reacções afectivas relacionadas com a exploração vocacional. Dos 54 itens, 53 dos quais são de resposta tipo likert (escala de cinco categorias de resposta, nos itens 1 a 43, e sete categorias, nos itens 44 a 53), e ainda um item (item 54) para indicar o número de domínios vocacionais explorados. A cotação de cada subescala do CES é obtida adicionando-se o valor de cada uma das respostas aos itens que a compõem, variando as pontuações mínimas e máximas de uma para a outra subescala, conforme o número de itens e a escala de resposta em causa. Os valores mais elevados traduzem a existência de crenças e reacções mais positivas à exploração e maior activação do processo exploratório (Taveira, 1997).

\section{Aplicação do tratamento}

A modalidade de tratamento foi a consulta psicológica em grupo. A selecção da modalidade de tratamento foi negociada com o cliente e os pais, explicando-se os efeitos e vantagens da aplicação da mesma.

A consulta psicológica em grupo favorece o desenvolvimento pessoal e social dos adolescentes. Ajuda os clientes a definir os seus planos de carreira e a prosseguir nas metas, bem como dedica atenção ao cliente e promove a construção de apoio emocional. Oferece oportunidades de exploração individual avaliadora do self que ajudam a atingir as metas, os planos de carreira ou as estratégias de decisão, assim como oferece informação a todos os participantes de um mesmo grupo para avaliar cuidadosamente 
as competências, a formação requerida e os resultados obtidos em diferentes percursos de carreira. Inclui, ainda, o uso de modelos de comportamento que demonstram como alcançar um plano de carreira e produz resultados desejados com uma duração de cinco sessões (Silva, 2004; Spokane \& Oliver, 1983; Taveira, 2001; Whiston et al., 1998).

Além disso, já segundo Schachter (1966), o tratamento em grupo ajuda a lidar com situações que geram ansiedade, como é o caso da tomada de decisão que os alunos têm que realizar no final do 9o ano de escolaridade. A pertença ao grupo promove, ainda, a criação de uma identidade pessoal e social na comparação com os outros, em que surgem as opiniões, os valores, as representações sociais e até mesmo as competências necessárias à exploração do self essencial ao processo de tomada de decisão de carreira. Esse processo de autoredefinição levará a novos comportamentos e atitudes (Leyens, 1979) que poderão ter conseqüências nas decisões escolares e profissionais.

Nesse sentido, o tratamento sob modalidade de consulta psicológica em grupo estruturouse em cinco momentos: (a) sessão pré-teste; (b) sessão inicial com a família; (c) sessões de consulta psicológica; (d) sessão final com a família e (e) sessão pós-teste.

A sessão pré-teste ocorreu duas semanas antes do início do tratamento. Ressalta-se que incluiu uma sessão inicial de avaliação pré-teste com o cliente.

A sessão inicial com a família decorreu uma semana antes do início do tratamento. Nessa sessão, foi realizada uma entrevista semiestruturada inicial com os pais do cliente (Pinto \& Soares, 2001), de forma a ajudar os pais a identificarem o sentido das suas acções conjuntas com o filho, a nelas reconhecerem objectivos ou intenções e, eventualmente, promover a confrontação. Essa sessão inicial tem a duração aproximada de 30 minutos, e tem como objectivo abrir vias à mudança de atitudes e comportamentos em função de novos objectivos partilhados com o filho (Pinto \& Soares, 2001). As técnicas usadas foram a reflexão acerca das opções e planos vocacionais dos filhos e o apoio na focalização em redes de ajuda e de suporte como facilitadores dos planos vocacionais dos filhos.

As sessões de consulta psicológica foram realizadas em cinco sessões de 90 minutos cada, de consulta psicológica em grupo (6-8 participantes), breve e estruturada. A primeira sessão destina-se a iniciar e a estruturar a relação de consulta com os clientes, a ajudálos na definição/clarificação na decisão específica que estão a enfrentar bem como na discussão dos seus pensamentos pessoais, teorias e crenças acerca da carreira e na identificação das suas dificuldades de tomada de decisão. São também elementos dessa sessão a revisão dos objectivos pessoais de cada aluno para a consulta psicológica, a sua história escolar, as suas aspirações e o desenvolvimento de um plano de tratamento individual. A informação geral acerca da história de tratamento vocacional de cada estudante também é recolhida.

Para atingir os objectivos dessa sessão, foram usadas técnicas tais como: exercícios escritos de reflexão a respeito do desenvolvimento vocacional e do processo de tomada de decisão, oferta de informação, análise lógica (analisar os problemas logicamente, oferecer oportunidades de análises e reestruturar o sistema de falsas lógicas), disputa de crenças irracionais e metáforas ou parábolas. As sessões dois e três têm como objectivos 
promover e guiar a exploração da carreira em profundidade, a fim de realçar a hipótese a testar e o compromisso com as próximas possibilidades de desenvolvimento de carreira. As oportunidades específicas de exploração das crenças de auto-eficácia, as expectativas do resultado, os valores e os interesses em relação aos papéis de vida e às actividades são também criadas nessas sessões.

A avaliação e o teste da listagem ordenada de opções preferidas são realizados, assim como são discutidos os recursos percebidos e as barreiras para alcançar o sucesso na acção. Usou-se a interpretação de testes, inventários e listas de verificação acerca do self, exercícios escritos de integração da informação acerca do self e exploração das alternativas, bem como feedback individualizado acerca dos planos vocacionais e projectos futuros. A quarta sessão visa a promover o progresso no acordo e a declaração pública de uma escolha. Discutem-se as aproximações à acção e realiza-se uma simulação da implementação da escolha através da oferta de informação prática referente ao mundo de trabalho e em opções vocacionais específicas (salário, oportunidades, actividades de trabalho), do uso de exercícios escritos de criação de uma lista de opções e de criação de alternativas semelhantes e de técnicas de role-playing e de relaxamento.

Por último, a sessão cinco propõe-se rever o processo de tratamento com cada cliente, elaborar conclusões sobre o processo e os resultados de tomada de decisão da carreira e fechar o relacionamento através de actividades de: ensaio através da imaginação das etapas necessárias à execução do plano, feedback individualizado referente às estratégias de tomada de decisão vocacional e aos planos vocacionais e projectos futuros e de avanços, e estimulação aberta de sentimentos e fecho da relação.

A sessão final com a família realizou-se uma semana após o término do tratamento. Teve como objectivo prestar esclarecimentos e aconselhamentos aos pais do cliente. As técnicas utilizadas foram o feedback individualizado referente aos resultados alcançados e a reflexão na criação da ajuda para as opções e os planos vocacionais dos filhos. Por último, a sessão pós-teste foi realizada duas semanas após o término do tratamento.

\section{Resultados e discussão}

\section{Avaliação da eficácia do tratamento}

No presente caso, pode afirmar-se que o tratamento foi eficaz, já que as metas estabelecidas foram alcançadas. Os resultados das medidas de pré e pós teste são apresentados na Tabela 1, que mostra a melhoria da qualidade dos resultados do cliente através dos distintos parâmetros das medidas de resultados usadas, o que reflecte a evolução do cliente do início para o fim do tratamento.

Como se pode concluir pela leitura da Tabela 1, relativamente aos níveis de indecisão de carreira, verifica-se que estes são baixos e inferiores ao ponto médio antes do tratamento. Contudo, as análises de estatística não paramétrica, através do teste de sinais, permitem-nos registrar a existência de diferenças estatisticamente significativas entre os momentos pré e pós-teste. No momento pós tratamento, o cliente registrou níveis mais baixos de indecisão de carreira, o que significa que a intervenção surtiu efeito no nível da indecisão.

No que respeita as medidas de exploração de carreira, antes do tratamento, no domínio das crenças associadas à exploração, registram- 
se, por um lado, valores de freqüência acima do ponto médio da escala, no valor instrumental atribuído às actividades de exploração orientada para o meio e para o self e na importância atribuída aos objectivos vocacionais, condições todas elas favoráveis ao envolvimento na exploração vocacional (Taveira, 2000).

Tabela 1. Resultado das medidas de pré e pós teste.

\begin{tabular}{llcc}
\hline Medidas & Ponto médio & Pré - teste & Pós - teste \\
\hline CDS - Indecisão de carreira & 42 & 32 & 22 \\
CES - Crenças de exploração & & & \\
Estatuto do emprego & 9 & 8 & 15 \\
Certeza nos resultados da exploração & 9 & 9 & 13 \\
Instrumentalidade externa & 33 & 35 & 50 \\
Instrumentalidade interna & 12 & 17 & 17 \\
Importância de obter a posição preferida & 9 & 12 & 13 \\
CES - Processo de exploração & & & \\
Exploração orientada para o meio & 15 & 7 & 12 \\
Exploração orientada para o self & 12 & 4 & 16 \\
Exploração intencional sistemática & 6 & 9 & 23 \\
Quantidade de informação obtida & 9 & 20 & 13 \\
CES - Reacções à exploração & & & 13 \\
Satisfação com a informação obtida & 9 & 8 & 8 \\
Stress na exploração & 12 & 19 & \\
Stress na tomada de decisão & 15 & 23 & \\
\hline
\end{tabular}

Por outro lado, registram-se valores abaixo do ponto médio da escala nas percepções acerca do estatuto de emprego e valores iguais ao ponto médio nas expectativas de resultado no domínio da carreira. Esse resultado é menos favorável ao envolvimento na exploração vocacional. Além disso, quando as expectativas de resultado baixas estão associadas a níveis elevados de importância atribuída aos objectivos vocacionais, como é o caso, podem originar níveis elevados e não desejáveis de ansiedade face à exploração e à decisão (cf. Taveira, 1997). Com efeito, verifica-se um baixo envolvimento do cliente na exploração orientada para o meio e menor envolvimento na exploração orientada para o self (valores abaixo do ponto médio da escala), níveis elevados de informação vocacional e valores altos na intencionalidade e carácter sistemático da conduta exploratória. Ao mesmo tempo, esse cliente não se apresenta satisfeito com a informação vocacional obtida até ao momento, e, tal como poderia ser esperado, apresenta níveis elevados de ansiedade face à exploração e ao compromisso vocacional.

Após o tratamento, registra-se a existência de diferenças estatisticamente significativas nos resultados de exploração de carreira. Verifica-se uma variação no sentido do aumento em todas as dimensões do processo de exploração de carreira bem como em todas, menos uma (instrumentalidade interna), nas dimensões das crenças de exploração avaliadas.

Na subescala satisfação com a informação obtida, a variação dos resultados também sofreu um aumento no sentido desejado. Por seu lado, as subescalas stress na exploração e stress na tomada 
de decisão, a variação de resultados foi no sentido desejado, da diminuição. Deve-se referir que a ansiedade face à tomada de decisão diminuiu para valores inferiores ao do ponto médio da escala. Assim, o cliente mudou em um sentido positivo na maioria das medidas entre os momentos de pré e pós teste. Além disso, o cliente activou diversos comportamentos de exploração específicos. Obteve informação sobre diversas áreas da carreira e desenvolveu uma lista de potenciais planos de carreira. No final, foi capaz de escolher a melhor alternativa. Nesse sentido, podemos afirmar que os resultados alcançados constituem um padrão de resultados muito positivo, reflectindo a eficácia do tratamento realizado.

Concluindo, o presente estudo, destinado a avaliar o efeito da intervenção psicológica vocacional na indecisão e na conduta exploratória, evidencia que a consulta psicológica vocacional produz efeitos positivos nos clientes. Os resultados obtidos através das medidas de pré e pós teste sugerem uma evolução no sentido favorável do nível de indecisão e de exploração vocacional, o que vai de encontro ao que seria esperado, de acordo com a teoria e a investigação empírica da indecisão e da exploração vocacional (cf. Taveira, 2000). Os resultados do estudo corroboram, também, os resultados dos estudos empíricos conduzidos para testar os efeitos das intervenções vocacionais breves (c.f. Silva, 2004; Spokane, 1991, 2004). Assim, os profissionais de orientação possuem bases empíricas consistentes para continuar a apostar na consulta psicológica breve estruturada, a fim de ajudar os jovens a apoiar as dificuldades na tomada de decisão vocacional.

Maria Céu Taveira Castro Silva Brás Cunha*

Doutora em Psicologia da Educação, docente do Departamento de Psicologia da Universidade do Minho Portugal.

\section{Liliana da Costa Faria}

Doutorada em Psicologia Vocacional, investigadora do Centro de Investigação em Psicologia, do Departamento de Psicologia da Universidade do Minho - Portugal.

E-mail: lilianafaria@delphis.com.pt

*Endereço para envio de correspondência:

Departamento de Psicologia. Campus de Gualtar. Universidade do Minho. 4710-057 Braga - Portugal

E-mail: ceuta@mail.telepac.pt 
Afonso, M. (2000). Exploração vocacional de jovens: condições do contexto relacionadas com o estatuto socioeconómico e com o sexo. Dissertação de Mestrado, Instituto de Educação e Psicologia, Universidade do Minho, Braga, Portugal.

Afonso, M., \& Taveira, M. C. (2001). Exploração vocacional de jovens. Condições do contexto relacionadas com o estatuto socioeconómico e com o género: Relatórios de investigação. Braga, Portugal: CEEP.

Archer, J. A., \& Lubinsky, D. (2003). Fostering exceptional development in intellectually talented populations. In W. B. Walsh (Ed.), Counseling psychology and optimal human functioning (pp. 25-54). Mahwah, NJ: Erlbaum.

Armstrong, P. I., \& Rounds, J. B. (2008). Vocational psychology and individual differences. In S.D. Brown \& R. W. Lent (Eds.), Handbook of counseling psychology (pp. 375-391). New Jersey: John Wiley \& Sons.

Berlyne, D. E. (1960). Conflict, arousal, and curiosity. New York: McGraw Hill.

Bowlby, J. (1969). Attachment and loss: Attachment. New York: Basic.

Brown, S. D., \& Krane, N. E. (2000). Four (or five) sessions and a cloud of dust: Old assumptions and new observations about career counseling. In S. D. Brown \& R. W. Lent (Eds.), Handbook of counseling psychology (pp. 740-749). New York: John Wiley \& Sons.

Brown, S. D., \& McPartland, E. B. (2005). Career interventions: Current status and future directions. In W. B. Walsh \& $\mathrm{M}$. L. Savickas (Eds.), Handbook of vocational psychology (pp. 195- 216). Hillsdale, NJ: Erlbaum.

Buela-Casal, G., \& Sánchez, A. (2002). Trastornos del sueño. Madrid: Síntesis.

Buela-Casal, G., \& J. C. Sierra (2002). Normas para la redacción de casos clínicos. Revista Internacional de Psicología Clínica y de la Salud/International Journal of Clinical and Health Psychology, 2, 525-532.

Buela-Casal, G., \& Sierra, J. C. (2001). Manual de evaluación y tratamientos psicológicos. Madrid: Biblioteca Nueva.

Chartrand, J. M., \& Robbins, S. B. (1997). Career factors inventory applications and technical guide. Palo Alto, CA: Consulting Psychologists Press.

Chartrand, J. M., Robbins, S. B., \& Morrill, W. H. (1994). The career factors inventory. Palo Alto, CA: Consulting Psychologists Press.
Cohen, J. (1988). Statistical power analysis for the behavioral sciences (2nd ed.). Hillsdale, NJ: Lawrence Earlbaum.

Crites, J. O. (1969). Vocational psychology. New York: McGrawHill.

Erikson, E. (1968). Identidad, juventud y crisis. México: Fondo de Cultura Económica.

Gati, I. (2006). Facets of career decision-making difficulties. British Journal of Guidance and Counselling, 34(4), 483-503,

Gati, I., Kraus, M., \& Osipow, S. (1996). A taxonomy of difficulties in career decision making. Journal of Counseling Psychology, 43(4), 510-526.

Germeijs, V., \& De Boeck, P. (2003). Career indecision: Three factors from decision theory. Journal of Vocational Behavior, $62(1), 11-25$

Gibson, R. C., \& Mitchell, M. H. (1998). Introduction to counseling and guidance. New Jersey: Prentice Hall.

Ginzberg, Z. (1951). Occupational choice: An approach to general theory. New York: Columbia University Press.

Gottfredson, L. S. (1981). Circumscription and compromise: A developmental theory of occupational aspirations. Journal of Counselling Psychology, (Monograph), 28.

Greenhaus, J. H., \& Connolly, T. F. (1982). An investigation of career exploration among graduates business students. Journal of College Student Personnel, 23, 314-319.

Gysbers, N. C., \& Moore, E. (1987). Career counselling. Skills and techniques for practitioners. Englewood Cliffs, NJ: Prentice-Hall.

Hill, C. E., Carter, J. A., \& O'Farrell, M. K. (1983). A case study of the process and outcome of time-limited counseling. Journal of Counseling Psychology, 30, 3-18.

Holland, J. L. (1997). Making vocational choices: A theory of vocational personalities and work environments (3rd ed.). Odessa, FL: PAR.

Jordan, J. P., \& Super, D. E. (1974). The predition of early adult vocational behavior. In D. F. Ricks, A. Thomas, \& M. Roff (Eds.), Life history research in psychopathology (pp. 108-130). Minneapolis, MN: University of Minnesota Press.

Kelly, K. R., \& Lee, W. C. (2002). Mapping the domain of career decision problems. Journal of Vocational Behavior, $61,302-326$ 
Krumboltz, J. D., \& Thoresen, C. E. (1964). The effect of behavioral counseling in group and individual settings on information-seeking behavior. Journal of Counseling Psychology, 11(4), 324-335.

Lent, R. W., \& Brown, S. D. (1996). Social cognitive approach to career development: An overview. Career Development Quarterly, 44, 310-321.

Leyens, J. P. (1979). Psicologia social. Lisboa: Edições 70.

Miller, M. J., \& Brown, S. D. (2005). Counseling for career choice: Implications for improving interventions and for working with diverse populations. In S. D. Brown \& R. W. Lent (Eds.), Career development and counseling: Putting theory and research to work. New York: Wiley \& Sons.

Montero, I., \& León, O. G. (2002). Clasificación y descripción de las metodologías de investigación en psicología. Revista Internacional de Psicología Clínica y de la Salud/International Journal of Clinical and Health Psychology, 2, 503-508.

Oliver, L. W., \& Spokane, A. R. (1988). Career-intervention outcome: What contributes to client gain? Journal of Counseling Psychology, 35, 447-462.

Osipow, S. H., Carney, C. G., Winer, J. L., Yanico, B., \& Koshier, M. (1976). The Career Decision Scale (3rd revision). Columbus, $\mathrm{OH}$ : Marathon Consulting \& Press.

Parsons, F. (1909). Choosing a vocation. Boston: HoughtonMifflin.

Peterson, G. W., Sampson, J. P., Jr., Lenz, J. G., \& Reardon, R. C. (2002). Becoming career problem solvers and decision makers: A cognitive information processing approach. In S. D. Brown (Ed.), Career choice and development (pp. 312-369). San Francisco, CA: Jossey-Bass.

Pinto, H. R., \& Soares, M. C. (2001). Influência parental na carreira: evolução de perspectivas na teoria, na investigação e na prática. Psychologica, 26, 135-149.

Rassin, E., \& Muris, P. (2005). Indecisiveness and the interpretation of ambiguous situations. Personality and Individual Differences, 39(7), 1285-1291.

Rounds, J. B. (1990). The comparative and combined utility of work value and interest data in career counseling with adults. Journal of Vocational Behavior, 37, 32-45.

Rounds, J. B., \& Tinsley, H. E. A. (1984). Diagnosis and treatment of vocational problems. In S. D. Brown \& R. W. Lent (Eds.), Handbook of counseling psychology (pp. 137-177). New York: Wiley.

Savickas, M. L. (1996). A framework for linking career theory and practice. In M. L. Savickas \& W. B. Walsh (Eds.), Handbook of career counseling theory and practice (pp. 191-208). Palo Alto, CA: Davies-Black.

Savickas, M. L. (1985). Identity in vocational development. Journal of Vocational Behavior, 27, 329-337.

Savickas, M. L. (2002). Career Construction: A developmental theory of vocational behavior. In D. Brown (Ed.), Career choice and development (4th ed., pp. 149-205). San Francisco, CA: Jossey- Bass.

Shimizu, K., Vondracek, F. W., Schulenberg, J. E., \& Hjostetler, M. (1988). The factor structure of the career decision scale: Similarities across selected studies. Journal of Vocational
Behavior, 32, 213-225.

Silva, J. T. (1997). Dimensões da indecisão da carreira: investigação com adolescentes. Dissertação de Doutoramento, Faculdade de Psicologia e Ciências da Educação da Universidade de Coimbra, Coimbra, Portugal.

Silva, J. T. (2004). A eficácia da intervenção vocacional em análise: implicações para a prática psicológica. In M. C. Taveira (Ed.), Desenvolvimento vocacional ao longo da vida. Fundamentos, princípios e orientações (pp. 95-125). Coimbra, Portugal: Editorial Almedina.

Spokane, A. R. (1991). Career intervention. Englewoods Cliffs, NJ: Prentice-Hall.

Spokane, A. R. (2004). Avaliação das intervenções de carreira. In L. M. Leitão (Ed.), Avaliação psicológica em orientação escolar e profissional (pp. 455-473). Coimbra, Portugal: Quarteto.

Spokane, A. R., \& Oliver, L. W. (1983). The outcomes of vocational intervention. In W. B. Walsh \& S. H Osipow (Eds.), Handbook of vocational psychology (pp. 99-126). Hillsdale, NJ: Lawrence Erlbaum.

Stumpf, S. A., Colarelli, S. M., \& Hartman, K. (1983). Development of the career exploration survey (CES). Journal of Vocational Behavior, 22, 191-226.

Sugalsky, T. D., \& Greenhaus, J. H. (1986). Career exploration and goal setting among managerial employees. Journal of Vocational Behavior, 29, 102-114.

Super, D. E. (1942). The dynamics of vocational adjustment. New York: Harper \& Row.

Super, D. E. (1957). The psychology of careers. New York: Harper \& Row.

Taveira, M. C. (1997). Exploração e desenvolvimento vocacional de jovens. Estudo sobre as relações entre a exploração, a identidade e a indecisão vocacional. Dissertação de Doutoramento, Instituto de Educação e Psicologia, Universidade do Minho, Braga, Portugal.

Taveira, M. C. (2000). Exploração vocacional: teoria, investigação e prática. Psychologica, 26, 5-27.

Taveira, M. C. (2001). O modelo de intervenção vocacional por programas. Lisboa: Casa Pia de Lisboa.

Taveira, M. C. (2005). Comportamento e desenvolvimento vocacional da adolescência. In M. C. Taveira, (Ed.), Temas de psicologia escolar. Uma proposta científico-pedagógica (pp. 143-178). Coimbra, Portugal: Quarteto.

Veerle, G., Karine, V., \& Bart, S. (2006).Indecisiveness and high school students' career decision-making process: Longitudinal associations and the mediational role of anxiety. Journal of Counseling Psychology, 53(4), 397-410.

Whiston, S. C., Brecheisen, B. K., \& Stephens, J. (2003). Does treatment modality affect career counseling effectiveness? Journal of Vocational Behavior, 62, 390-410.

Whiston, S., Sexton, T., \& Lasoff, D. (1998). Career-intervention outcome: A replication and extension of Oliver and Spokane (1988). Journal of Counseling Psychology, 45(2), 150-165.

Yost, E., \& Corbishley, M. (1987). Career counseling: A psychological approach. San Francisco, CA: Jossey-Bass. 